\title{
Geographical variation in Plasmodium vivax relapse
}

\author{
Katherine E Battle ${ }^{1 *}$, Markku S Karhunen ${ }^{1}$, Samir Bhatt ${ }^{1}$, Peter W Gething ${ }^{1}$, Rosalind E Howes ${ }^{1}$, Nick Golding ${ }^{1}$, \\ Thomas P Van Boeckel ${ }^{2}$, Jane P Messina ${ }^{1}$, G Dennis Shanks ${ }^{3}$, David L Smith ${ }^{4,5}$, J Kevin Baird ${ }^{6,7}$ and Simon I Hay ${ }^{1,5^{*}}$
}

\begin{abstract}
Background: Plasmodium vivax has the widest geographic distribution of the human malaria parasites and nearly 2.5 billion people live at risk of infection. The control of $P$. vivax in individuals and populations is complicated by its ability to relapse weeks to months after initial infection. Strains of $P$. vivax from different geographical areas are thought to exhibit varied relapse timings. In tropical regions strains relapse quickly (three to six weeks), whereas those in temperate regions do so more slowly (six to twelve months), but no comprehensive assessment of evidence has been conducted. Here observed patterns of relapse periodicity are used to generate predictions of relapse incidence within geographic regions representative of varying parasite transmission.
\end{abstract}

Methods: A global review of reports of $P$. vivax relapse in patients not treated with a radical cure was conducted. Records of time to first $P$. vivax relapse were positioned by geographic origin relative to expert opinion regions of relapse behaviour and epidemiological zones. Mixed-effects meta-analysis was conducted to determine which geographic classification best described the data, such that a description of the pattern of relapse periodicity within each region could be described. Model outputs of incidence and mean time to relapse were mapped to illustrate the global variation in relapse.

Results: Differences in relapse periodicity were best described by a historical geographic classification system used to describe malaria transmission zones based on areas sharing zoological and ecological features. Maps of incidence and time to relapse showed high relapse frequency to be predominant in tropical regions and prolonged relapse in temperate areas.

Conclusions: The results indicate that relapse periodicity varies systematically by geographic region and are categorized by nine global regions characterized by similar malaria transmission dynamics. This indicates that relapse may be an adaptation evolved to exploit seasonal changes in vector survival and therefore optimize transmission. Geographic patterns in $P$. vivax relapse are important to clinicians treating individual infections, epidemiologists trying to infer P. vivax burden, and public health officials trying to control and eliminate the disease in human populations.

Keywords: Malaria, Plasmodium vivax, Map, Relapse, Periodicity, Recurrence, Recrudescence, Strain

\section{Background}

Malaria is a significant global public health problem and the greatest burden of disease is found in the world's poorest countries [1]. The majority of malaria morbidity and mortality is caused by two of the five species of Plasmodium that naturally infect humans, Plasmodium falciparum and Plasmodium vivax. The broader global distribution of $P$. vivax relative to $P$. falciparum puts an estimated 2.5 billion people at risk for endemic vivax

\footnotetext{
*Correspondence: katherine.battle@zoo.ox.ac.uk; simon.hay@zoo.ox.ac.uk 'Department of Zoology, Spatial Ecology and Epidemiology Group, Tinbergen Building, University of Oxford, South Parks Road, Oxford, UK ${ }^{5}$ Fogarty International Center, National Institutes of Health, Bethesda, MD, USA Full list of author information is available at the end of the article
}

malaria [2,3]. An increasing body of evidence has shown that $P$. vivax should no longer be thought of as a benign and rarely fatal disease [4-9], but instead as being capable of causing severe disease and death, particularly in pregnant women and small children [9-12].

Plasmodium vivax is epidemiologically and biologically different to P. falciparum and it is not, therefore, appropriate to assume that control methods developed for falciparum malaria are directly transferable to $P$. vivax [13-16]. Biological features of $P$. vivax that distinguish it from $P$. falciparum also present unique challenges to the control of the parasite [17-19]; in elimination settings, $P$. vivax is often the "last parasite standing" following 
P. falciparum elimination [20,21]. Plasmodium vivax gametocytes are present earlier in the progression of a primary or recrudescent infection compared to $P$. falciparum $[17,22]$, such that the majority of patients have sufficient gametocytaemia to allow for transmission before diagnosis or treatment may occur [23-25]. In addition, $P$. vivax gametocytes are transmitted more efficiently to Anopheles mosquito vectors than those of $P$. falciparum and are transmissible at lower parasite densities $[18,26,27]$. Within the mosquito, $P$. vivax sporozoites develop faster than $P$. falciparum at equivalent temperatures, which contributes to its exploitation of a wider geographic range [28].

Perhaps the most epidemiologically important feature of $P$. vivax biology is its ability to relapse in the weeks and months following a primary parasitaemia via a dormant liver stage known as the hypnozoite [29-31]. This potential for long-term latency provides the obvious advantage of safe harbour during cold winter months when circulation in blood creates potential host immune system dangers without the benefit or opportunity for onward transmission. Therefore the term "infection" has various meanings for $P$. vivax. Infection may refer to the introduction and presence of parasites in the body, but with $P$. vivax, unlike $P$. falciparum, this can also refer to a symptom-less latent infection. The origin of renewed parasitaemia following a primary vivax infection or a "recurrence" is also ambiguous; it could be due to a hypnozoite-triggered relapse, a resurgence of erythrocytic parasites as a recrudescence, or an entirely new reinfection. See Figure 1 and Table 1 for a description of the pathways between types of infection and attack, and distinctions in terminology. The hypnozoite fundamentally distinguishes $P$. vivax from $P$. falciparum in almost every important biological, epidemiological, clinical, and public health respect.

The hypnozoite stage in the life cycle of $P$. vivax and the potential for relapse makes chemical therapies that target only the blood stage of infection ineffective as a radical cure. The 8-aminoquinolines are the only class of drugs known to have activity on the hypnozoite parasite [32-34]. Primaquine therapy, the only currently licensed radical cure, comes with caveats that add to the challenge of controlling the parasite to the point of elimination. Primaquine is associated with potentially fatal haemolysis in individuals with glucose-6-phosphate dehydrogenase (G6PD) deficiency $[32,35,36]$ and is contraindicated in pregnant women because of the risk of acute haemolytic anaemia in the foetus of unknown G6PD status [37]. The hypnozoite stage and the paucity of therapy for safe and effective treatment render vivax malaria an exceedingly difficult challenge for clinicians and those responsible for the control of endemic malaria. Relapse also has critical implications for understanding epidemiological metrics such as the basic reproduction number and force of infection, obtained from prevalence rates derived from malariometric surveys and cartographic studies that form a central part in elimination scenario planning $[38,39]$.

It has long been known that there is significant geographical variation in the rate at which a "strain" of $P$. vivax relapses [40-42]. Temperate and subtropical strains often exhibit either a long incubation or latent period (Figure 1) of around eight to ten months. Tropical strains are characterized by short incubation times and short latency (approximately three to six weeks) [43]. Incubation period refers to the time from sporozoite

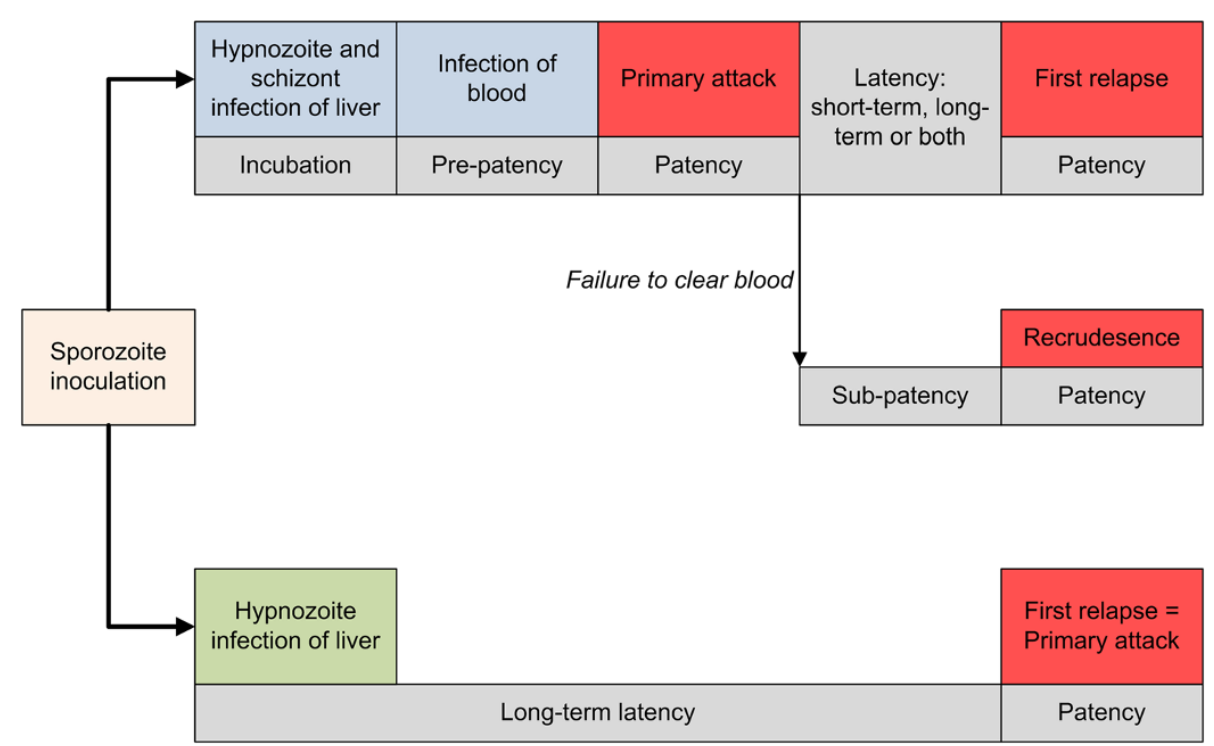

Figure 1 Pathways to infection of blood and clinical attacks in Plasmodium vivax malaria. 
Table 1 Glossary of terms relevant to Plasmodium vivax relapse

\begin{tabular}{|c|c|}
\hline Term & Definition \\
\hline Infection & Presence of parasites in any of its forms of incubation, prepatency, patency, subpatency or latency. \\
\hline Incubation & The period between inoculation of sporozoites and release of merozoites into the blood stream (primary exo-erythrocytic cycle). \\
\hline Prepatency & The period prior to a primary attack where asexual parasites in the blood are both not detectable and asymptomatic, though present. \\
\hline Patency & The period of clinical attack with demonstration of asexual parasites in blood as the cause of illness. \\
\hline Subpatency & The period following a primary attack where asexual parasites are both not detectable and asymptomatic, though present. \\
\hline Recrudescence & $\begin{array}{l}\text { An event following sub-patency when parasites are both demonstrated to be present and the cause of another clinical attack } \\
\text { or asymptomatic patency. }\end{array}$ \\
\hline Latency & $\begin{array}{l}\text { The period between a primary attack and a relapse; in some strains also the period between inoculation of sporozoites and the } \\
\text { occurrence of a patent primary attack, typically six months or more. }\end{array}$ \\
\hline Relapse & Patent asexual parasitaemia originating from activation of latent hypnozoites. \\
\hline Re-infection & Patency by asexual blood stage parasites deriving from a new inoculation of sporozoites. \\
\hline Recurrence & $\begin{array}{l}\text { A newly patent parasitaemia occurring at any point after clearance of sub-patency of a primary parasitaemia where the origin is } \\
\text { not known with certainty as being a reinfection, recrudescence or relapse. }\end{array}$ \\
\hline
\end{tabular}

Definitions are presented in chronological order of events.

inoculation (the mosquito bite) to the primary blood-stage infection. The latent period describes the time from the primary attack to relapse. How hypnozoite relapse is triggered, and the source of this phenotypic variation, is unresolved [44]. One theory is that the mechanism is an adaptive trait of the parasite to sequester or "hibernate" during times when climatic conditions would be inhospitable to the parasite's anopheline mosquito vectors [45-47]. Another is that latent hypnozoites are activated by a systemic febrile illness, explaining the large number of $P$. vivax relapses that follow P. falciparum infections [47-49]. These hypotheses need not be mutually exclusive.

Regardless of the triggering mechanism and aetiology of relapse, evidence from both controlled experimental and natural settings indicate considerable geographical variation in the timing of relapse. Although the historical perception has been that frequent relapsing strains originate from the tropics and long-latency strains from temperate regions [31], it does not sufficiently describe the observed variation in relapsing phenotypes. This binary classification conflicts with evidence of long-latency strains in tropical regions in the Americas, for instance. Coatney et al. [43] described in 1971 that there were three patterns of relapse. These included the Chesson strain of New Guinea-South Pacific which exhibits a short incubation period (seven to fourteen days), followed by regular re-invasions of the bloodstream within approximately three weeks after the primary infection and may continue to relapse for more than 18 months without a radical cure of the hypnozoite stage. The St Elizabeth strain from southern USA has a similar incubation period to the Chesson strain, but hypnozoites remain quiescent for several months following the primary infection before relapsing at regular intervals of three to four weeks for up to two years [43]. A third variety, such as the strain once found in the Netherlands [42], has a long period of incubation (around eight months) before the primary clinical episode followed by frequent relapses (the lower arm of Figure 1). This three-type classification likely oversimplifies the degree of variation in $P$. vivax relapse periodicity and offers limited information regarding the geographic origin of the described phenotypes. Furthermore, the majority of $P$. vivax strains have short incubation periods and the greatest difference lies in the latency period from primary attack to first relapse.

Geographic zones have been proposed for distinguishing areas with similar timing and frequency of $P$. vivax relapse following sporozoite inoculation. Figure $2 \mathrm{~A}$, adapted from White [47], and modified by the boundaries of the malaria endemicity map proposed by Lysenko and Semashko (for the maximum range of malaria circa 1900) [50], illustrates a proposed distribution of relapse phenotypes. White noted the historical perception that strains that relapse quickly originate from Southeast Asia. Temperate and subtropical areas are characterized by long-latency strains. The Indian subcontinent and South America are thought to contain both long-latency and frequent relapsing strains. A second geographic description of variation in time to relapse is described in a recent study by Lover and Coker [51]. The authors analysed the time to relapse in experimentally infected individuals relative to the geographic origin of the strain. They found that, overall, temperate strains relapse more slowly than tropical ones. However, they also found that New World tropical strains relapse more slowly than Old World tropical strains, and Eurasian temperate strains relapse more slowly than temperate strains from the Western Hemisphere [51]. Figure 2B illustrates this implied classification system.

While the maps in Figure 2 were derived from observations of relapse phenotype, others have grouped regions based on similar ecological and epidemiological 


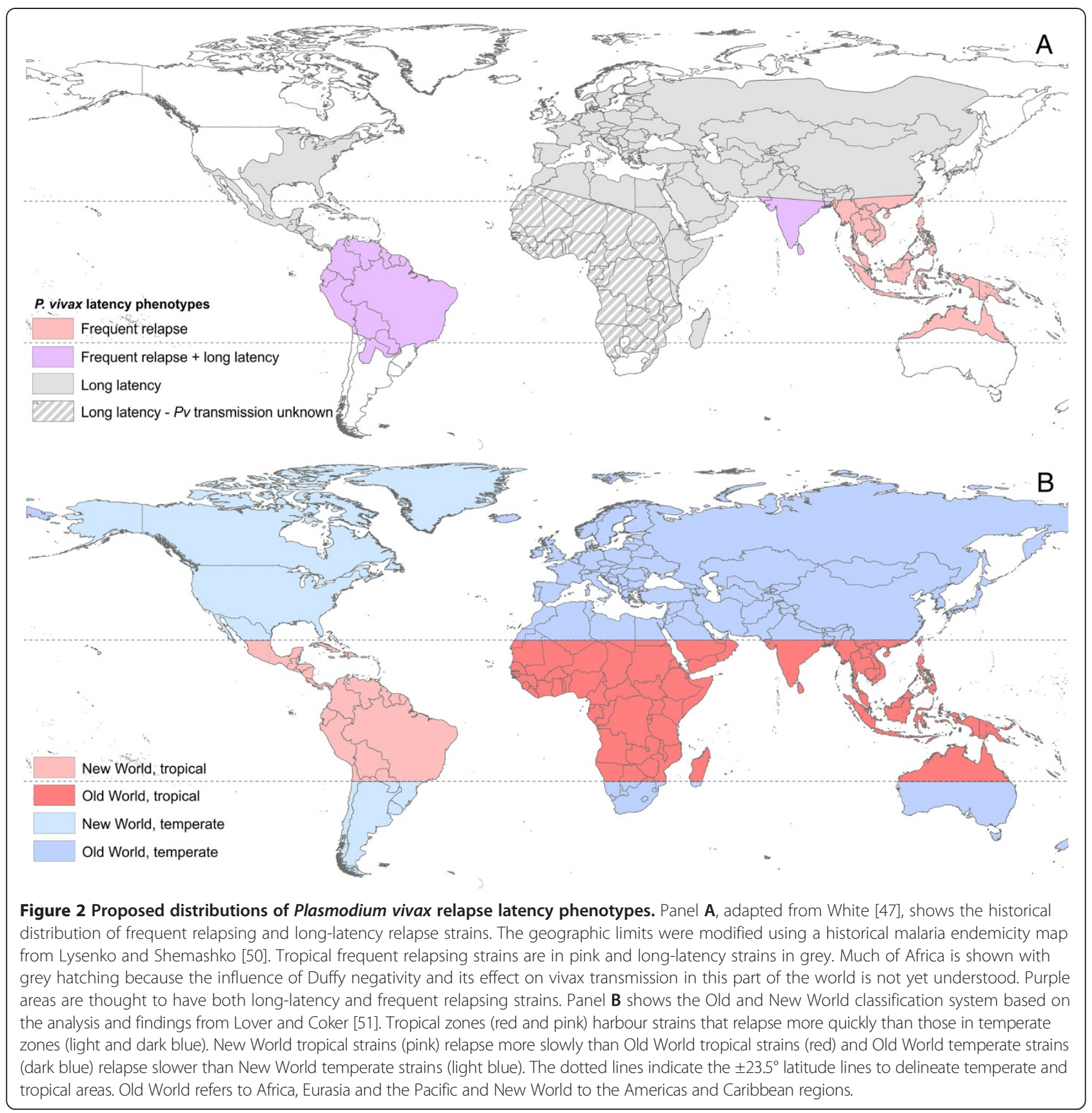

characteristics in the absence of relapse observations. These regions may reveal patterns of relapse and help elucidate determinants of the periodicity observed. The zones proposed by Macdonald [52] are shown in Figure 3. They are described by Macdonald as "zoogeographical" malaria regions and share commonalities with many historical biogeographical zonations [53-55]. The approximate boundaries of the zones are delineated by climatic variables that influence malaria transmission rates, such as temperature and rainfall, the intensity of transmission observed, as well as the abundance and behaviours of the locally dominant vector species [52].
A systematic review of $P$. vivax relapse events was conducted with the aim of revealing systematic geographical patterns of relapse frequency and a quantitative description of the potential time to relapse in different regions of the $P$. vivax-endemic world.

\section{Methods}

\section{Data assembly}

A formal literature search for data was conducted on PubMed [56] with the keywords: "vivax AND relapse" on 15 November, 2012 and updated on 24 October, 2013. The search returned 449 references. This list was 


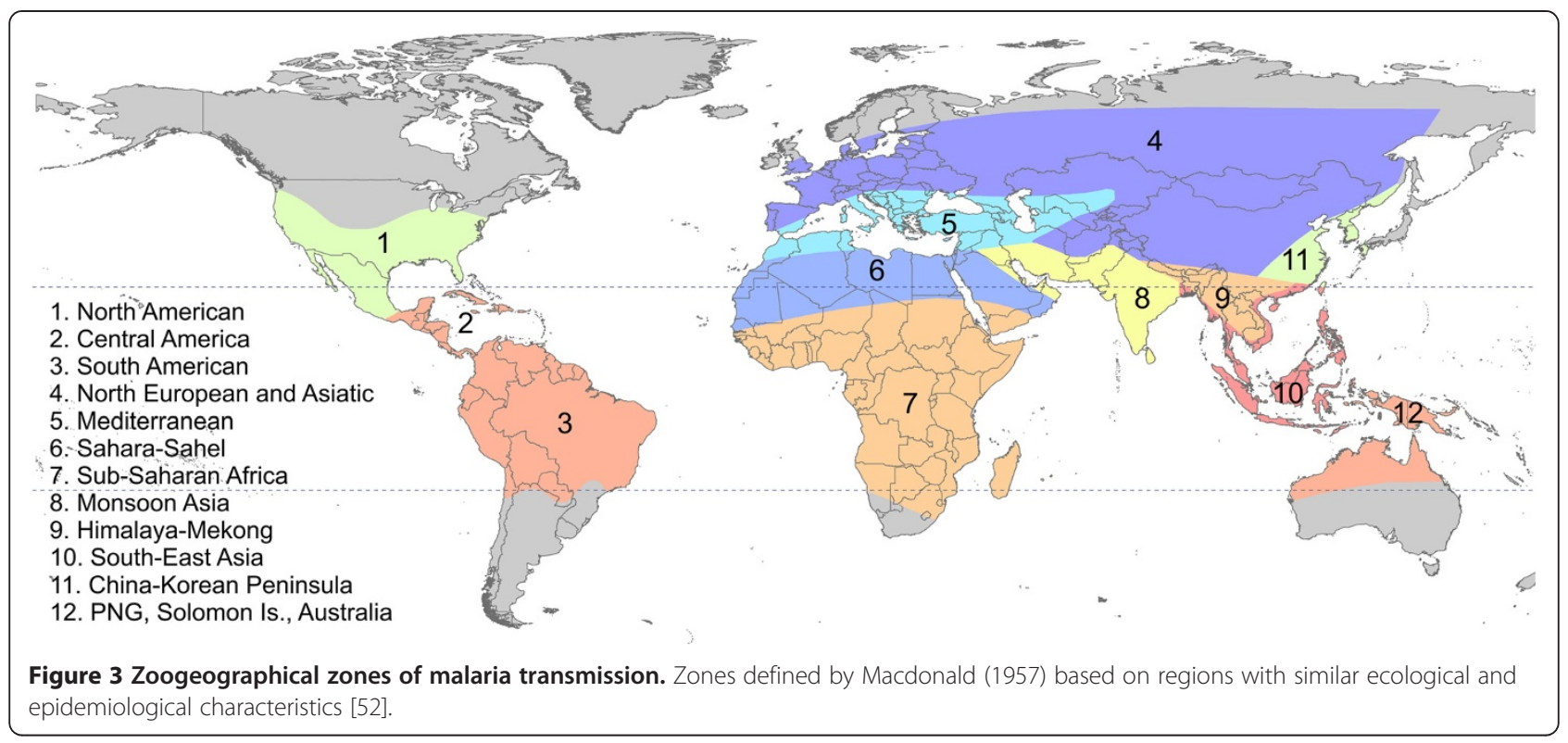

augmented with the reports of $P$. vivax relapse cited by Baird and Hoffman [32] and from the reference list of two recent reviews of variation in relapse periodicity $[47,51]$. Additional studies were obtained by correspondence with colleagues active in this research area. Malaria in the military was also examined using references from the US Army and medical records from British soldiers who contracted malaria during duty in World War II. Medical records were obtained from contacts and the Malaria Research Library, now the Malaria Reference Library, kept at the London School of Hygiene and Tropical Medicine. The aim was to obtain as much data as possible regarding $P$. vivax recurrence in all regions where vivax malaria is or has been endemic. The literature sources ostensibly refer to the recurrence events as relapses; however treatment trials and studies conducted in endemic areas may include recrudescences and reinfections in measures of relapse. Because much of the data on $P$. vivax relapse in patients not treated with primaquine originated before use of the drug became common following World War II [57], no restrictions were applied on study dates.

The exclusion criteria for the studies were minimal. Data were not used from patients who had been treated with a sufficient dose (15 mg per $\mathrm{kg}$ for 7 or 14 days) of primaquine, or any 8-aminoquinoline drug (pamaquine, plasmochin or pentaquine), due to the effect of the drug on hypnozoites, and therefore patterns of relapse. For example, a series of clinical trials demonstrated that 8aminoquinoline drug failures (relapses) occurred 6090 days post-patency, whereas untreated relapses almost always occurred between day 17 and day 35 postpatency [58]. Studies that had treated patients with a five-day course of $15 \mathrm{mg}$ base of primaquine or less, which was shown to be ineffective in preventing relapse [59], were permitted. Relapse in patients treated with a seven-day course of primaquine in South America, where the treatment schedule has been shown to be inadequate [60], were also considered. Blood-stage treatments were not exclusion criteria, but were noted for analysis purposes. Mefloquine prophylaxis and treatments such as mepacrine (quinacrine, atabrine) or chloroquine may cause a delay in relapse because the drugs retain suppressive levels in the patient for long periods after treatment [61,62], making it difficult to distinguish the observed relapse as a first or second relapse. A 14-day cutoff was applied to data abstracted from drug treatment trials. Any re-appearance of infection before the 14th day was categorized as a treatment failure, while infection after day 14 was listed as a relapse. This conservative cut-off was applied to maximize sensitivity. A primary relapse is unlikely to occur before two weeks, even in fast relapsing strains [63]. However, a 14-day cut-off may result in some recrudescence events being classed as a relapse.

When possible, data on time to recurrence from the start of treatment of the primary infection were abstracted to the individual level. Start of treatment was almost invariably the first day of patency, and we considered it most probable that the vast majority of recurrences represented relapses. The majority of studies reported by the day, but those that reported the week or month of relapse were also included (with the last day of the month or week given as the time to relapse). Studies that aggregated months together were excluded. The period of follow-up was recorded for all individuals in each study, including those that did not experience a relapse. Data on the type of patient (prison volunteer, malaria therapy patients, soldiers, etc.) were also collected 
as it is likely that this would have influenced the time to first relapse. Data on duration of prepatent period from studies performed in experimental settings were recorded where this information was available.

\section{Georeferencing}

Geopositioning of relapse studies was implemented using established methods [64] for those references that did not provide specific coordinates for the study site. The latitude and longitude of entries that provided cities or towns were located as points $(<10 \mathrm{sq} \mathrm{km})$ using Google Maps [65]. The centroid of small and large polygons $(>25$ to $<100 \mathrm{sq}$ $\mathrm{km}$ and $>100 \mathrm{sq} \mathrm{km}$, respectively), such as islands, regions or countries were obtained using ArcMAP 10.0 [66] to determine the latitude and longitude of those areas. The latitude and longitude values recorded corresponded to the origin of infection. Therefore, infections in returning travellers were geopositioned to the place of travel and malaria therapy or experimental trials were positioned to the origin of the strain used.

\section{Statistical analysis}

The number of cases, total person time observed, and mean and standard deviation of time to first relapse were calculated among individuals who experienced at least one relapse in each study. The incidence rate of relapse was calculated from the number of relapse events and total person time. The factors affecting this rate were modelled using mixed-effects meta-analysis in the package metafor $[67,68]$ within the statistical programming language $R$ [69]. As data must be normalized for use in metafor, how to best accomplish this was tested by applying different transformations to the data from each study and assessing deviation from normality by the Shapiro-Wilk test.

The geographic zones described above (Figures 2 and 3 ) were included in the meta-analysis of relapse rate as categorical moderators. These included the three phenotypic zones shown in White (hereafter referred to as the White-

Table 2 Comparison of geographic classification systems

\begin{tabular}{lllllll}
\hline System & $\mathbf{T}^{\mathbf{2}}$ & $\mathbf{I}^{\mathbf{2}}$ & $\mathbf{H}^{\mathbf{2}}$ & $\mathbf{R}^{\mathbf{2}}$ & $\mathbf{A I C}$ & $\mathbf{A I C c}$ \\
\hline White-3 & 1.3 & 97.1 & 34.0 & $33.6 \%$ & 707.7 & 707.9 \\
Lover & 1.6 & 97.5 & 40.8 & $19.4 \%$ & 728.4 & 729.0 \\
White-5 & 0.9 & 95.4 & 21.8 & $57.3 \%$ & 623.6 & 624.0 \\
Macdonald & 0.8 & 95.0 & 20.0 & $59.9 \%$ & 612.7 & 614.1 \\
Modified Macdonald & 0.8 & 95.0 & 19.9 & $60.1 \%$ & 612.9 & 614.0
\end{tabular}

Mixed-effects meta-analysis has been performed for 214 different studies or study arms by using the R package metafor [68]. The statistics are: $\tau^{2}$, amount of residual heterogeneity; $I^{2}$, residual heterogeneity/unaccounted variability; $\mathrm{H}^{2}$, unaccounted variability/sampling variability; $\mathrm{R}^{2}$, calculated from the residual heterogeneity $\left(\mathrm{T}^{2}\right)$ and the residual heterogeneity of an empty model as suggested by Raudenbush [70]; AIC, Akaike information criterion; and AICC, corrected AIC. The values of AIC, BIC and AICC are based on the restricted maximum likelihood, as this corresponds to the REML (restricted maximum likelihood) estimator recommended by experts [67].
3 system), which were also shown differentiated by Old World and New World (White-5), the four zones described by Lover and Coker, and lastly the 12 zoogeographical regions as described by Macdonald [47,51,52]. Model choice was performed between these geographic systems using information criteria given by metafor (see Table 2). For the best geographic system obtained from this, a meta-analysis of mean time to first relapse among patients with observed relapse events was performed. See Additional file 1 for more information regarding model choice and the meta-analysis. Kaplan-Meier survival curves were generated from pooled individual data from each zone. These curves are intended to illustrate the observed qualitative patterns of relapse in each zone.

\section{Relapse maps}

To visualize geographic variation in relapse, maps were generated by plotting points of median time to relapse in individuals who experienced a relapse from each study included in the final dataset. Regional maps were produced to illustrate the relapse incidence and mean time to relapse modelled within the geographic system chosen in the meta-analysis.

\section{Results}

\section{Data assembly}

Following the literature search and collection of unpublished sources, 121 references were found to contain data on time to first recurrence in patients not treated with a sufficient radical cure. Further details regarding the results of the literature review are found in Additional file 1. Details and summary statistics for the 121 references showing time to first recurrence are shown in Additional file 2. Of those, 87 references reported data at the individual level with time to relapse reported in days or values less than or equal to one month (Figure 4). The resulting dataset contained information on 30,049 individuals, of whom 5,731 experienced at least one recurrence. These data are provided in Additional file 3. The observed recurrences are most likely to be relapses, but, in probably rare instances, recrudescences may also be represented among data classified as early relapse ( $<60$ days). The list of references included in the final database is available in Additional file 4.

Relapse rate data were available from 29 different countries and regions. The vast majority of the data were from India (78\%, 23537/30049); although of the patients to experience a relapse, only one third $(34 \%, 1931 / 5731)$ originated from the subcontinent (Additional file 1). There were data from 23 known strains in experimental infections, but the majority of individuals contracted wild $P$. vivax $(94 \%, 28149 / 30049)$. Many of the subjects were not residents of endemic areas. For example, over one third of the patients to relapse $(37 \%, 2731 / 5731)$ were 


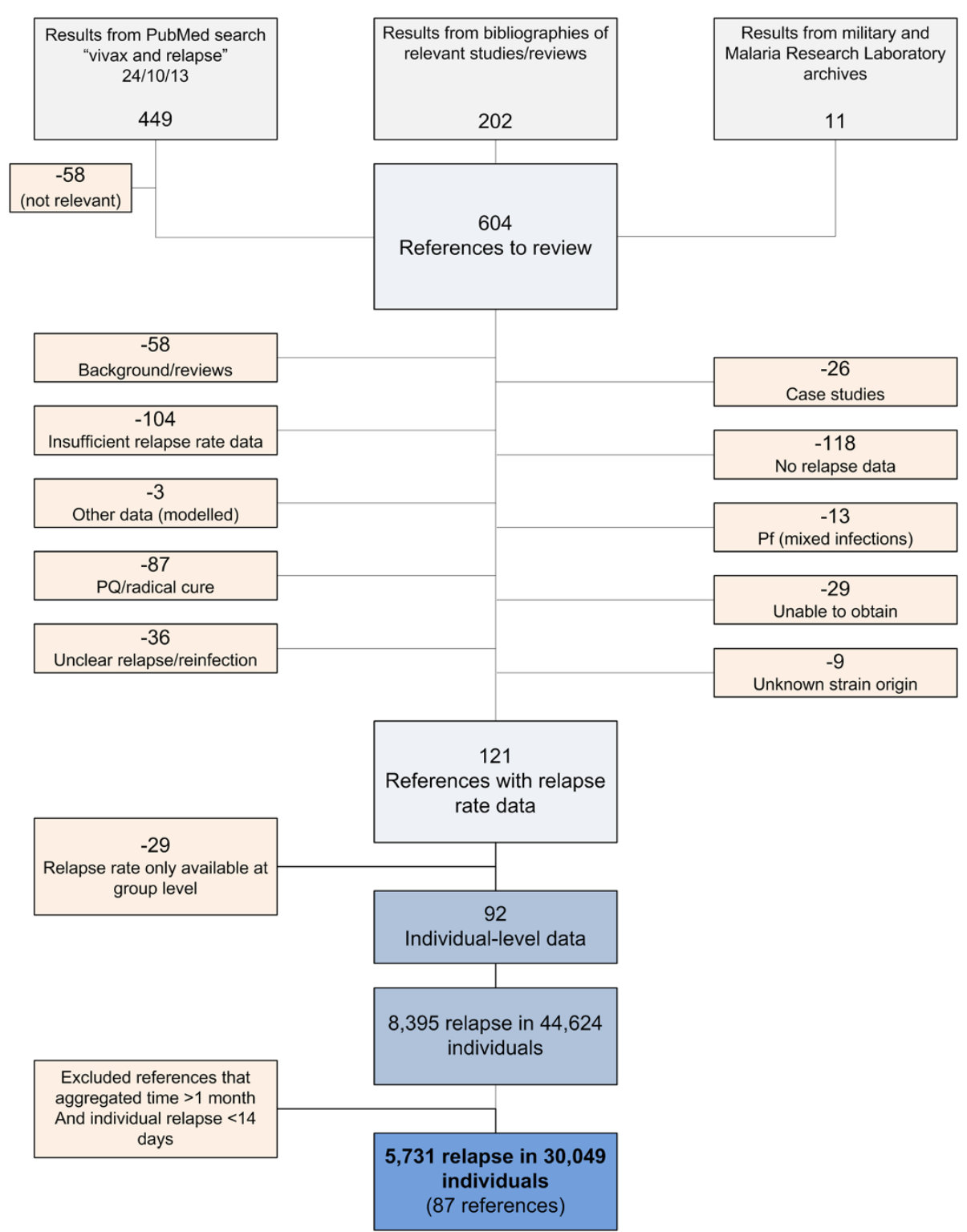

Figure 4 Schematic overview of the literature review procedure and results to obtain individual records of relapse and follow-up.

military personnel deployed from non-endemic regions. A summary of key aspects of the dataset, such as treatment and patient type, is available in Additional file 1.

\section{Statistical analysis}

Regardless of the transformation applied to the raw data for each study (identity, log, square root or FreemanTukey [71]), the time to first relapse deviated significantly from normal (plots shown in Additional file 1). The deviation was smallest under the log-transformation, which is common for incidence-rate data [72]. The model choice analysis was therefore carried out using log-transformed data. The Macdonald classification system yielded the best description of the data, judging by pseudo- $\mathrm{R}^{2}$, the Akaike information criterion (AIC) and corrected AIC (AICc, which accounts for small sample sizes, see Table 2). Possible ways to simplify the Macdonald system such that geographically contiguous zones with similar transmission suitability would be combined were assessed. Further details are provided in Additional file 1. Combining zones 4 and 11 slightly improved the model fit. Zones 5 and 6 , and 9 and 10, respectively, were also combined as there were no relapse data available from zones 6 and 9. The revised zones are shown in Figure 5A.

Table 3 presents two estimates of relapse incidence rate for the modified Macdonald system as the number of first relapses per 100,000 person days. One is the crude estimate based on raw data, and the other is the 


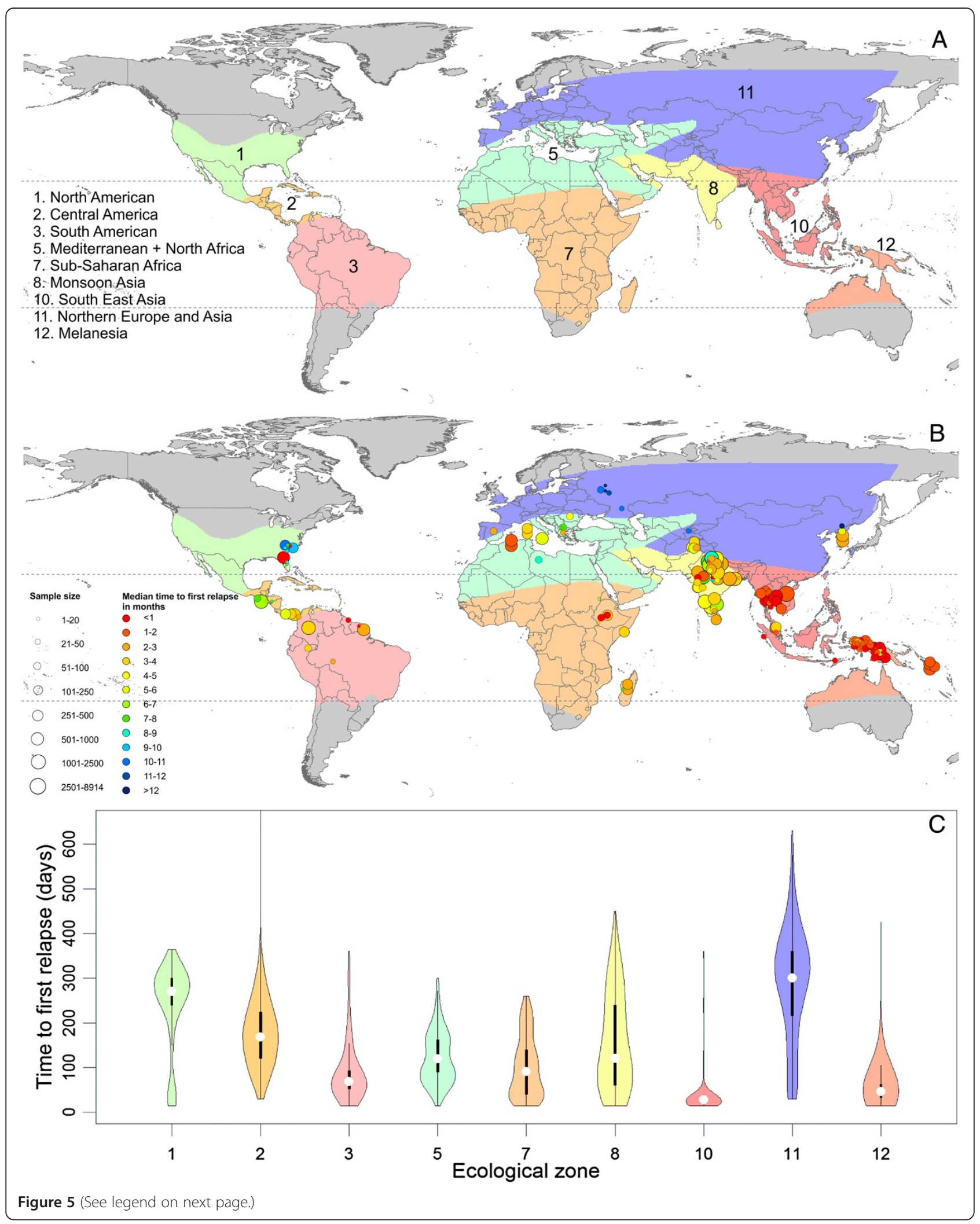


(See figure on previous page.)

Figure $\mathbf{5}$ Revised zoogeographical zones and observed time to first relapse. Panel $\mathbf{A}$ illustrates the revised zoogeographical zones used to describe the time to first relapse. Panel $\mathbf{B}$ shows the median observed time to relapse in each study used to obtain individual data. The size of each point varies by sample size and the time to first relapse is shown on a spectrum of red (less than one month) to dark blue ( $>12$ months). Violin plots in Panel $\mathbf{C}$ show the observed time to first relapse in individuals from each zone in Panels $\mathbf{A}$ and $\mathbf{B}$. The coloured areas correspond to each zone and to a smoothed approximation of the frequency distribution (a kernel density plot) of the time relapse within each geographic region. The black central bar represents the interquartile range and the white circles indicate the median values. Note that the maximum value for zone 2 extends beyond the plot.

result obtained from the meta-analysis. The highest observed and predicted incidence values are found in zones $9+10$ and 12, corresponding to Southeast Asia and Papua New Guinea (PNG) plus the Solomon Islands (Melanesia). It is predicted that there will be approximately $800-1,200$ relapses per 100,000 person days in this part of the world, compared with the estimated 130 relapses in northern Asia and Europe (zones 4 and 11). The crude and predicted estimates are very different for some zones (namely 3 and 8 ). This is because the mixed-effect meta-analysis attributes unusually low and high case numbers to inter-study variation and these do not contribute substantively to the rate estimate. A relevant incidence measure could not be calculated for zone 8 (India) because the observed data included several large studies ( $>2,000$ patients) in which the majority of patients did not experience a relapse (Additional file 1).

Table 4 shows the raw and modelled estimates of mean relapse time for each of the geographic zones in the modified Macdonald system. These were obtained by running a meta-analysis for mean time to relapse and its standard deviation within each study (in a separate analysis from the original run for the incidence rates), and these are calculated from only the observed relapse events. This produces a modelled estimate of relapse time that is based on

Table 3 Relapse incidence rates for the modified Macdonald system

\begin{tabular}{|c|c|c|}
\hline Ecological zone & $\begin{array}{l}\text { Based on raw data, } \\
\text { ML with } 95 \% \mathrm{Cl}\end{array}$ & $\begin{array}{l}\text { Model-based, } \\
\text { REML with } 95 \% \mathrm{Cl}\end{array}$ \\
\hline 1 & 357 (Cl: 318-400) & 455 (Cl: 313-662) \\
\hline 2 & 217 (Cl: 198-238) & 259 (Cl: 120-557) \\
\hline 3 & 419 (Cl: 328-528) & 1093 (Cl: 535-2,233) \\
\hline $5+6$ & 214 (Cl: 186-245) & 262 (Cl: 82-839) \\
\hline 7 & 221 (Cl: 191-255) & 213 (Cl: 95-477) \\
\hline 8 & $25(\mathrm{Cl}: 24-26)$ & $62(\mathrm{Cl}: 33-116)$ \\
\hline $9+10$ & 975 (Cl: 811-1163) & 836 (Cl: 351-1,995) \\
\hline $11+4$ & 138 (Cl: 120-159) & 134 (Cl: 64-278) \\
\hline 12 & 1023 (Cl: 981-1,067) & 1224 (Cl: 689-2,174) \\
\hline \multicolumn{3}{|c|}{$\begin{array}{l}\text { The numbers are presented as first relapses per } 100,000 \text { person days. The } \\
\text { estimate based on raw data is obtained by dividing the number of relapses by } \\
\text { follow-up time and using Ulm's exact formula [73] for confidence intervals. The } \\
\text { model-based estimates are calculated from the results obtained from meta- } \\
\text { analysis performed by the R package metafor. ML refers to the maximum } \\
\text { likelihood and REML to restricted maximum likelihood. }\end{array}$} \\
\hline
\end{tabular}

the least variable data sources. The Indian zone figures are therefore more plausible in these results. The modelled results show the fastest times to relapse are found in Southeast Asia (zone 9+10) and Melanesia (zone 12), around 45 days. South America (zone 3) also had a rapid time to relapse (65 days). However, there were relatively few records from zone 3 (Figure 5B) and some of the heterogeneity in relapse patterns may have been missed. Zone 1, North America, is predicted to have relapse times of about six months. Central America, zone 2, is estimated to have a relapse time of five to six months, driven by a few studies with long relapse intervals observed in Mexico [74]. The Mediterranean zone $(5+6)$, a region of seasonal transmission, has a modelled mean time to relapse of five months. Based on the raw data, the mean time to relapse from the few data points in the sub-Saharan Africa zone (7) was only one month. Finally, the northern Europe and Asia zone $(11+4)$ has by far the longest modelled mean time to relapse of ten months.

Finally, Figure 6 presents the survival curves for the modified Macdonald system. Note that the meta-analysis models described above do not yield survival curves. The curves in Figure 6 are based on the Kaplan-Meier survival function estimator, and they are calculated from pooled raw data within the geographic zones to provide a quantitative comparison of relapse patterns among zones. For

Table 4 Mean time to relapse among geographic zones

\begin{tabular}{lll}
\hline Ecological zone & $\begin{array}{l}\text { Based on raw data, } \\
\text { ML with 95\% Cl }\end{array}$ & $\begin{array}{l}\text { Model-based, } \\
\text { REML with 95\% Cl }\end{array}$ \\
\hline 1 & $100(\mathrm{Cl}: 99-101)$ & $185(\mathrm{Cl}: 162-208)$ \\
2 & $239(\mathrm{Cl}: 239-240)$ & $164(\mathrm{Cl}: 117-212)$ \\
3 & $53(\mathrm{Cl}: 53-53)$ & $65(\mathrm{Cl}: 18-113)$ \\
$5+6$ & $153(\mathrm{Cl}: 153-153)$ & $151(\mathrm{Cl}: 80-221)$ \\
7 & $31(\mathrm{Cl}: 30-31)$ & $107(\mathrm{Cl}: 57-158)$ \\
8 & $181(\mathrm{Cl}: 180-181)$ & $120(\mathrm{Cl}: 82-159)$ \\
$9+10$ & $289(\mathrm{Cl}: 288-290)$ & $41(\mathrm{Cl}:-11-92)$ \\
$11+4$ & $89(\mathrm{Cl}: 87-90)$ & $299(\mathrm{Cl}: 254-345)$ \\
12 & $122(\mathrm{Cl}: 121-123)$ & $47(\mathrm{Cl}: 12-81)$ \\
\hline
\end{tabular}

The model-based estimates have been calculated by using the $\mathrm{R}$ package metafor, which acknowledges interstudy variation. Thus the numbers differ from raw means calculated from the data. ML refers to the maximum likelihood and REML to restricted maximum likelihood. Please note that sample means by the very definition concern only observed events, and consequently this table ignores person time from censored observations. 


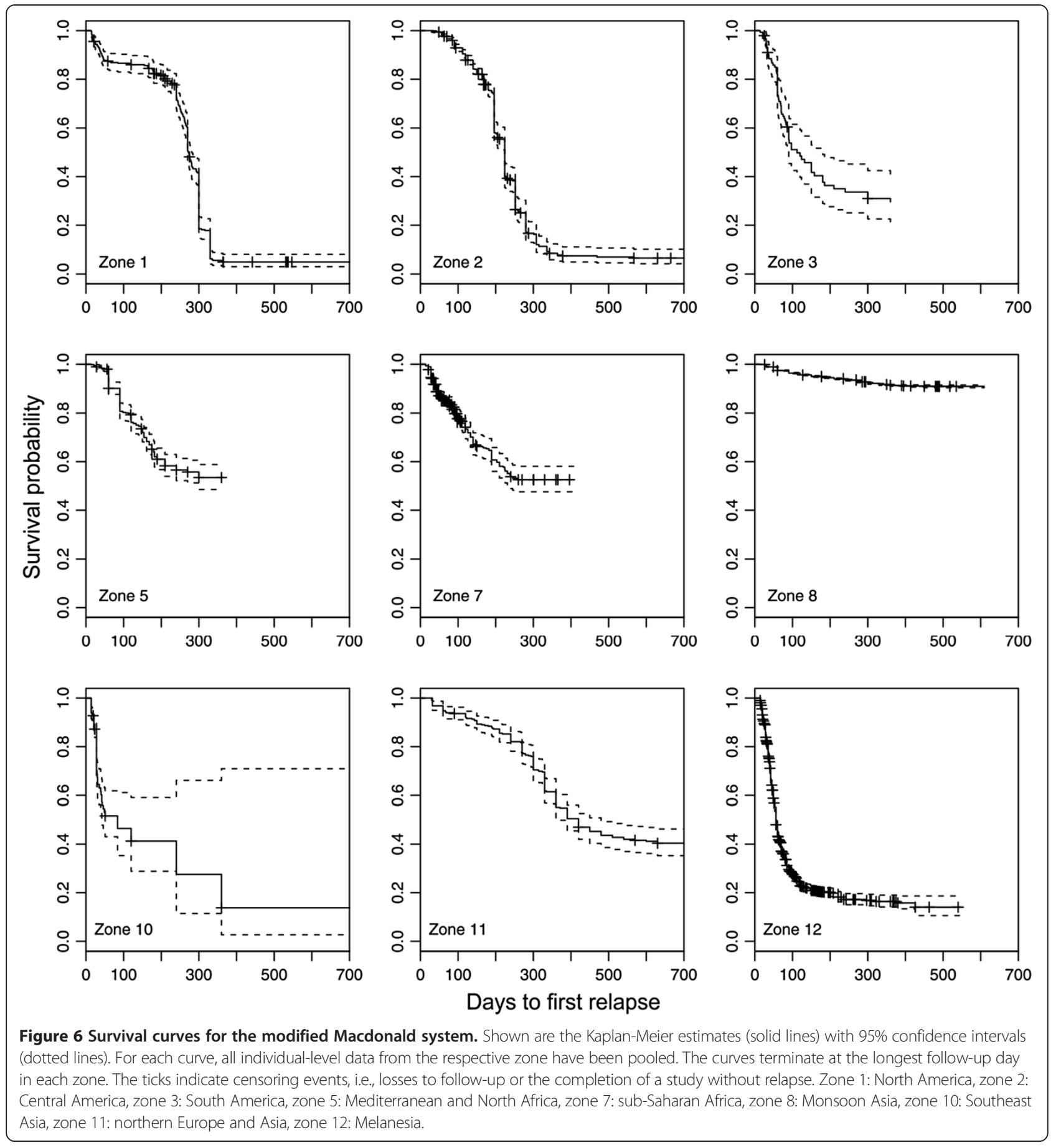

example, in zone 1 , the majority of the patients observed had relapsed by day 300 , whereas in zone 12 , most patients had relapsed before day 100 . The curve in zone 8 does not reveal much regarding the time to relapse because of the small number of relapsing patients.

\section{Relapse maps}

The revised zoogeographical zones used in the analyses described above are shown in Figure 5A. In Figure 5B the median time to relapse for study locations is specified with points inside the geographic zones. The map illustrates a concentration of fast-relapsing strains in Southeast Asia and Melanesia. The heterogeneity in relapse periodicity observed in zone 8 is also apparent in this map. The variation in the North American zone is due to the behaviour of strains occasionally relapsing quickly after a long incubation period between inoculation and primary attack (data available from experimental 
inoculations only), but for the most part relapses followed a long period of latency after a short incubation period. Summary statistics of the time to relapse by zone are shown in Additional file 1. Panel $\mathrm{C}$ in Figure 5 is a violin plot of observed time to relapse in each zone. This illustrates that those shorter relapses in North America are fairly rare. The violin plots also show that the longer periods to relapse in Central America and Southeast Asia are rare. Heterogeneity in other zones, such as 8 and $11+$ 4 , is also demonstrated by the violin plots. Figure 7 illustrates the results of the predicted relapse incidence and mean time to relapse by region. In Figure 7A, zone 8 is hatched out as the resulting estimate is biologically implausible and is most likely caused by the handful of studies with large numbers of patients not reporting relapse during the observation period.

\section{Discussion}

The aims of this paper were to review the timing and frequency of $P$. vivax relapse of known origin in patients not treated with a hypnozoitocide to characterize variance in these patterns within geographic dimension, to identify a system to classify the variation in relapse observed and to describe the pattern of relapse in each area. A modified classification of the zoogeographical zones of malaria transmission outlined by Macdonald [52] was found to best describe the observed variation in relapse incidence. The rate of relapse and mean time from primary infection to relapse was predicted in each of the nine zones. These quantitative estimates of the contribution of relapses to $P$. vivax case incidence are crucial in informing estimates of disease burden and the origin of acute attack, i.e., from biting mosquitoes or

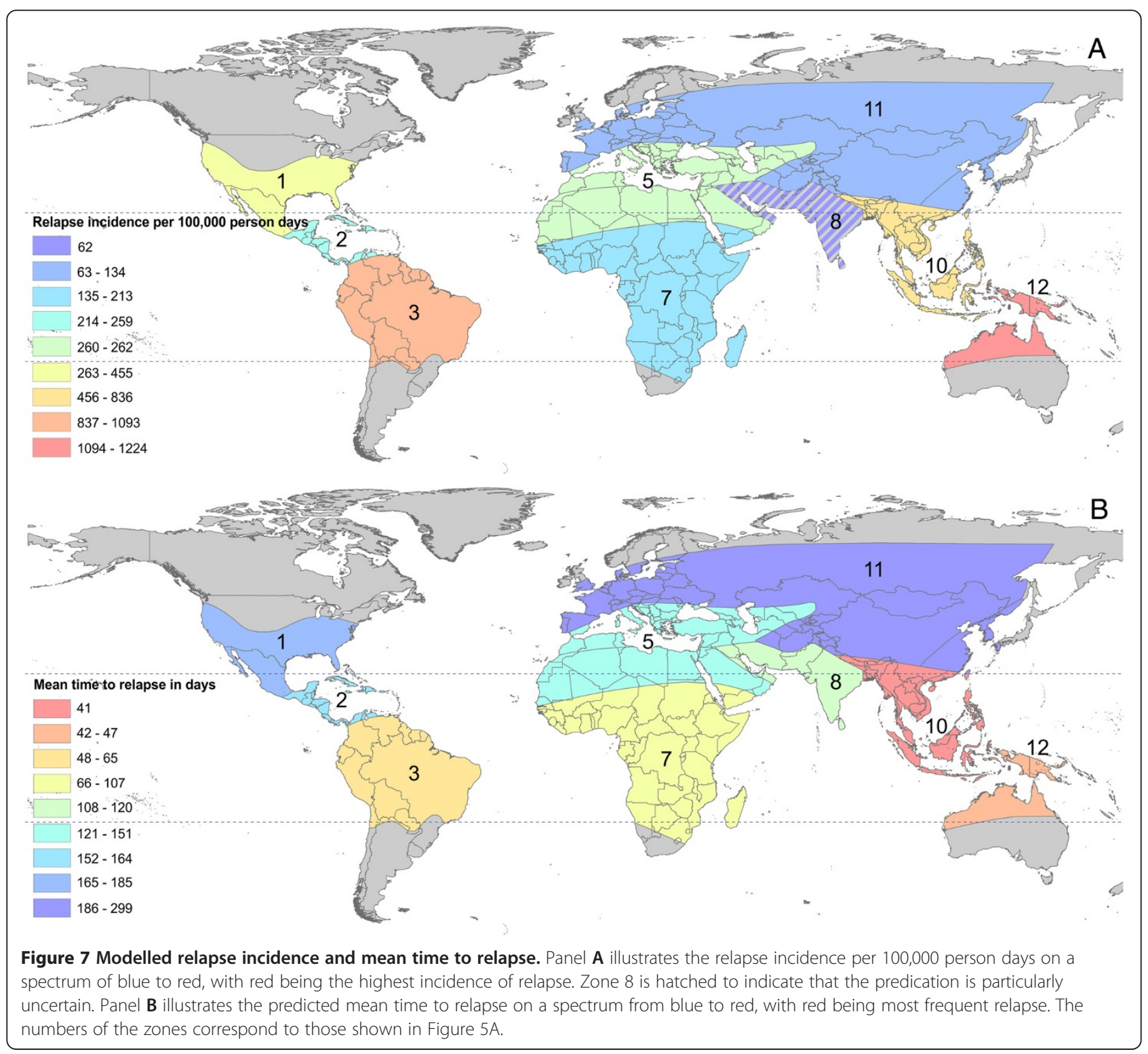


emergent hypnozoites. This understanding, in turn, informs critical decision-making in control strategies that effectively weigh the benefit of anti-mosquito versus hypnozoitocidal interventions. They also help identify regions in which strains have long-term latency and are therefore undetectable to standard diagnostic methods (rapid diagnostic tests and microscopy) for long periods of time.

The results presented here further refine historical interpretations and recent analyses of the geographic variation in relapse periodicity. As shown in Figure 2, tropical strains relapse more rapidly than temperate strains and New World strains vary from those in the Old World. White's illustration of the variation in relapse phenotype shown in Figure 2A shows that Southeast Asia and Asia-Pacific is the only region having exclusively frequent relapse behaviour. The results also showed infections from this region to relapse quickly, with a few rare exceptions. White showed that both frequent relapsing and long-latency strains are present in India and South America. The data from India appear to affirm this, with relapse patterns from the subcontinent and surrounding areas so heterogeneous that it was impossible to generate logical model predictions of relapse incidence for the region. The low incidence in region 8 shown in Table 3 is not believed to be a reflection of the presence of long relapsing strains, but rather a result of natural infections that either did not result in a relapse or the resulting parasitaemia was too low to be detected by the study. There is not presently an explanation for the lack of relapses, but this phenomenon has also been observed in recent tafenoquine trials in India [75]. The variation in relapse timing in the raw data observed in India (Figure $5 \mathrm{~B}$ ) is likely a result of the wide variation in transmission settings found within this zone. There are tropical forest areas, similar to zone 12, dry habitats like those in zone 5 and highland areas that border zone 11. In addition, the presence of Anopheles stephensi, adapted to breeding in artificial water collections [76,77], has extended transmission into urban areas. Therefore, in addition to issues of data availability and study design, the range of ecological settings in the zone, and likely some of the other zones, may also contribute to the variance in relapse behaviours observed.

The results presented for South America were different from what was shown in White's phenotype map (Figure 2A). South America was predicted to have a high relapse incidence, comparable to Southeast Asia, and a two-month mean time from primary attack to relapse. The data available from this region were limited and determining the cause for the observed difference is therefore difficult. There has been renewed interest in the origin of the American strains of $P$. vivax, whether they originate from somewhere in Asia or were sent west from
Africa by migration and the slave trade, as has been proposed for P. falciparum [78]. This could influence the nature of the relapse periodicity observed. Improved understanding of the phylogeny of $P$. vivax may reveal information about the pattern of relapse in this region.

The analysis by Lover and Coker [51] revealed that tropical regions relapse more quickly than temperate strains. However, Central America and sub-Saharan Africa had relapse patterns similar to the Mediterranean with moderate relapse incidence (around 250 relapses per 100,000 person days) and five to six months between primary infection and relapse. These regions seem to be better described as an "intermediate" relapsing phenotype between the frequent relapsing strains in Southeast Asia and South America and the long-latency temperate strains in North America and Europe/Asia.

The results of the northern temperate regions concur with the findings of Lover and Coker [51]. The authors noted that while in general, temperate strains relapse more slowly than tropical strains and that New World tropical strains were slower than Old World strains, the opposite was true of the temperate strains. Based on this analysis, the New World temperate strains relapsed more rapidly than the Old World temperate strains (Figure 7). The modelled results showed that the relapse incidence was 455 per 100,000 person days and mean time to relapse was six months in North America. However, again, this high incidence compared to that in northern Asia and Europe (134 relapses per 100,000 person days) could be due to a few exceptional experimental subjects who received large sporozoite inoculations [79-81].

The utility of the predictions made is limited by the nature of the data available. There are few contemporary data on $P$. vivax infections in patients not treated with a hypnozoitocide. Therefore, much of the data used were from drug trials on adult workers, military personnel and prison "volunteers", as well as data from when malaria was used to treat neurosyphilis patients. The age and immunity of the patients would perhaps not be representative of relapse as it would occur among residents of the strain region of origin. This could be important because the children in many endemic settings likely carry the greatest burden of relapsing infections [82]. Some experimental challenge subjects were inoculated with relatively heavy sporozoite dosages, a factor that greatly influences the time from primary attack to relapse [47] and is likely to differ from relapses following more modest numbers of sporozoites acquired from wild anophelines. It is straightforward to attribute an infection as a relapse or re-infection in experimental settings, but this could not be distinguished for $P$. vivax infections acquired in the wild. Effort was made to obtain studies where the follow-up period was conducted in a non-endemic area (for example, in a hospital in a city). 
Of the 5731 records of relapse $88 \%(n=5030)$ were obtained from experimental or non-local populations (such as military personnel). Lastly, the strains used in therapy and drug trials in the first half of the twentieth century were of "known" origin and infections were geopositioned to those sites. However, it is not certain if the strains used were of their named origin. For example, the "Madagascar" strain was obtained from an Indian seaman whose last port of call was in Madagascar [83] and may conceivably, therefore, originate from elsewhere.

A principal limitation of this work is the inability to conclusively identify a recurrent infection as a relapse, recrudesence or reinfection. This is particularly an issue given the rising rate of resistance to standard treatments such as chloroquine [84]. In cases of chloroquine failure, recurrence can occur one to two months after initial treatment [63], making differentiation between relapse and recrudescence a challenge. Chloroquine and chloroquinecombination therapies were by far the most common treatment regimens $(82 \%, 24787 / 30049)$. Of those patients to receive chloroquine, 2519 patients experienced a relapse or recurrence, 523 of which occurred before 60 days. This is equivalent to $9 \%(523 / 5731)$ of the relapse records. In addition, the effect of resistance on recurrence is likely abated by the historical nature of the dataset. The first cases of chloroquine-resistant $P$. vivax were reported in 1989 [85]. Of the 5731 records of relapse, 2080 occurred before 60 days and $82 \%$ of those $(n=1701)$ were observed before 1989. Therefore, increased resistance is unlikely to have a large effect on the results and instances of recrudesence being classed as a relapse would have been rare. While the relapse signal represented in these data certainly contains some noise due to reinfection or recrudescence, we considered these other sources of recurrences improbable relative to relapse. Figures $5 \mathrm{C}$ and 6 seem consistent with this assumption because recurrence due to reinfection or recrudescence would have been far more stochastic than relapse, obscuring or effacing the patterns shown by the randomness of timing of those events relative to primary parasitaemia.
There are aspects of the data that were not incorporated into the analysis performed here that could be addressed in future work. First, it was difficult to account for strains with long incubation periods before primary infection (information only available for a subset of data from experimental settings) followed by relatively short time to relapse. This was occasionally exhibited by the North American St Elizabeth strain and P. vivax multinucleatum from China (see Additional file 3). The link between the sporozoite dose and latency, mentioned above, was shown in the literature to be an important factor in determining relapse patterns $[46,86,87]$, but was not incorporated into the analysis as it cannot be known for wild infections. This may be a possible explanation why the incidence of relapse in North America was greater than that predicted for Central America. However, this was likely not a common problem with the experimental studies used. The majority of studies aimed to induce patency and the sporozoite inoculations were large, but not extreme. There were only a handful of studies included that used particularly large inoculations in order to study the effect of dosage on relapse pattern [79-81].

The type of patient varied among studies (prison volunteers, military personnel, malaria therapy patients, outpatients in an endemic area, etc.) and the drug type and dosage varied within and among studies. In some studies, primary attacks were treated with insufficient doses of 8aminoquinolines or drugs that have long half-lives. Mepacrine has a half-life of up to a month and can delay relapses by about 30 days $[88,89]$ and chloroquine, the most common drug in the dataset, can delay parasite reappearance by anywhere from two to six weeks [47]. Inclusion of the subject-type and treatment as explanatory variables was tested; however, the results were similar to the simpler model used (see Additional file 1). Finally, the analysis only addressed the periodicity between primary attack and first observed recurrence (relapse). The modelled estimates of incidence do not account for multiple relapses. Both the frequency and number of relapses will vary based on a variety of factors including inoculums, age

Table 5 Strategies for modelling survival data obtained from many dissimilar sources

\begin{tabular}{|c|c|c|c|}
\hline Statistical method & Accounts for individual-level variation & $\begin{array}{l}\text { Accounts for } \\
\text { between-study } \\
\text { variation }\end{array}$ & R packages \\
\hline Fixed-effects meta-analysis & No; operates on summary statistics & No & Many software packages, e.g., meta and metafor \\
\hline Mixed-effects meta-analysis & No; operates on summary statistics & Yes & $\begin{array}{l}\text { Many packages, e.g., meta and metafor; } \\
\text { also general-purpose software such as } \\
\text { Ime4 may be used }\end{array}$ \\
\hline $\begin{array}{l}\text { Survival analysis for } \\
\text { pooled data }\end{array}$ & Yes & No & A number of packages, e.g., survival, eha and flexsurv \\
\hline $\begin{array}{l}\text { Survival analysis with } \\
\text { mixed effects }\end{array}$ & Yes & Yes & $\begin{array}{l}\text { Most notably } \mathrm{R} / \text { coxme; flexible software seems to } \\
\text { be hard to find }\end{array}$ \\
\hline
\end{tabular}

Data were analysed using mixed-effects meta-analysis, which is common for this type of study. All of the methods have strengths and weaknesses. 
of patient and origin of infection. While many studies did not follow patients long enough to report multiple relapses, further work in this area will be essential to obtain measures of the $P$. vivax force of infection.

In addition to the limitations posed by the data and survey study designs, the analysis is limited by the types of statistical methods available for this kind of task (see Table 5). It would be preferable to use a statistical model that is both hierarchical (to account for between-study variation) and employs a suitable survival-analysis likelihood. Unfortunately, software used to fit this type of model was numerically unstable and hence the mixedeffects meta-analysis was employed.

The mechanism of hypnozoite activation to cause an acute attack (relapse) remains unknown. There is clearly variation in relapse "phenotype". Based on the results presented here, timing of relapse appears to vary geographically in conjunction with areas of similar ecology and malaria transmission patterns. However, it is difficult to determine whether long latency occurs in regions of frequent relapse (tropical areas such as zones $9+10$ and 12). A long-latency relapse may be thought to be another short-term relapse in a succession of rapid relapses, and the genotype cannot reveal if it is in fact a separate relapse "event" [47]. Nonetheless, understanding broad patterns of relapse is of use epidemiologically. There tends to be fewer overall relapses in the long-latency strains because hepatocytes that host the hypnozoites may die before the relapse event occurs. The resulting burden of hypnozoites from different strains or regions has implications for sensitivity to primaquine and therefore the dosage of primaquine that should be used [47]. This was observed in treatment of soldiers returning from Korea (fewer hypnozoites) [90] relative to those returning from the Pacific (high hypnozoite burden) [58] and will have implications for future control strategies.

\section{Conclusions}

Frequency of relapse varies geographically. The association between relapse rate and the geographic regions does not clarify causation. Geographic variation does not directly imply environmental cues as triggers for relapse, even if the revised Macdonald system resembles the distribution of transmission suitability. Relapse frequency may result from evolved responses to average transmission season duration or arise from proximate cues, such as triggers from other infections, correlated with $P$. vivax transmission and/or vector suitability periods. There is likely an interaction between activation of latent hypnozoites from infection and an evolved trait for strains from areas of seasonal transmission to remain dormant during periods of low mosquito abundance. Regardless of the cause, these patterns are important for the treatment of individual infections, measures of $P$. vivax burden and the prospects for control and eventual elimination of the disease from endemic areas.

\section{Additional files}

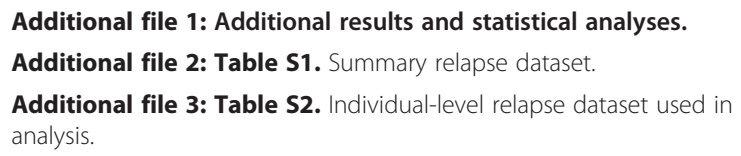

\section{Abbreviations}

95\% Cl: 95\% confidence interval; AIC: Akaike information criterion; AICc: Corrected Akaike information criterion; BIC: Bayesian information criterion; G6PD: Glucose-6-phosphate dehydrogenase; ML: Maximum likelihood; PNG: Papua New Guinea; REML: Restricted maximum likelihood.

\section{Competing interests}

The authors declare that they have no competing interests.

\section{Authors' contributions}

KEB and SIH conceived the study and oversaw its implementation with assistance from MSK, PWG and JKB. KEB wrote the first draft of the manuscript and assembled data with assistance from REH, JPM and GDS. Environmental data were provided by TPVB. MSK led the design of the modelling framework with input from NG, SB and DLS. All authors participated in the interpretation of results and in the writing and editing of the manuscript. All authors read and approved the final manuscript. KEB and $\mathrm{SIH}$ will act as guarantors for the paper.

\section{Acknowledgements}

We would like to thank Nick White, David Warhust, Ellis McKenzie, Wallace Peters, Bill Collins, Andrei Beljaev, John Barnwell and David Bradley for their input and suggestions at the early stages of this work. We acknowledge the contribution of references from Ric Price, Socrates Herrera, unpublished data contribution from François Nosten and Cindy Chu and data support from Lilia Gonazalez-Ceron and Manjoit Roy. We also thank David Pigott for proofreading.

This work was supported by a Wellcome Trust Senior Research Fellowship (\#095066) held by SIH, which also supports KEB, MSK and REH. PWG is a Medical Research Council Career Development Fellow (\#K00669X) and receives support from the Bill and Melinda Gates Foundation (\#OPP1068048) that also supports SB. NG is funded by a grant from the Bill \& Melinda Gates Foundation (\#OPP1053338). JPM is funded by the International Research Consortium on Dengue Risk Assessment Management and Surveillance (IDAMS, European Commission 7th Framework Programme (\#21803)). TPVB was funded by a grant from the Belgian Fond National pour la Recherche Scientifique and the Fondation Wiener-Anspach. DLS and SIH acknowledge funding support from the RAPIDD program of the Science \& Technology Directorate, Department of Homeland Security, and the Fogarty International Center, National Institutes of Health. JKB is supported by Wellcome Trust grant \#B9RJIXO.

\section{Author details}

${ }^{1}$ Department of Zoology, Spatial Ecology and Epidemiology Group, Tinbergen Building, University of Oxford, South Parks Road, Oxford, UK. ${ }^{2}$ Department of Ecology and Evolutionary Biology, Princeton University, Princeton, NJ, USA. ${ }^{3}$ Australian Army Malaria Institute, Enoggera, Queensland, Australia. ${ }^{4}$ Department of Epidemiology and Malaria Research Institute, Johns Hopkins Bloomberg School of Public Health, Baltimore, MD, USA. ${ }^{5}$ Fogarty International Center, National Institutes of Health, Bethesda, MD, USA. ${ }^{6}$ Eijkman-Oxford Clinical Research Unit, Jalan Diponegoro No 69, Jakarta, Indonesia. ${ }^{7}$ Nuffield Department of Medicine, Centre for Tropical Medicine, University of Oxford, Oxford, UK.

Received: 7 February 2014 Accepted: 31 March 2014 Published: 15 April 2014 
References

1. Sachs J, Malaney P: The economic and social burden of malaria. Nature 2002, 415:680-685

2. Gething PW, Elyazar IRF, Moyes CM, Smith DL, Battle KE, Guerra CA, Patil AP, Tatem AJ, Howes RE, Myers MF, George DB, Horby P, Wertheim HFL, Price RN, Mueller I, Baird JK, Hay SI: A long neglected world malaria map: Plasmodium vivax endemicity in 2010. PLoS Negl Trop Dis 2012, 6:e1814

3. Battle KE, Gething PW, Elyazar IR, Moyes CL, Sinka ME, Howes RE, Guerra CA, Price RN, Baird KJ, Hay SI: The global public health significance of Plasmodium vivax. Adv Parasitol 2012, 80:1-111.

4. Anstey NM, Russell B, Yeo TW, Price RN: The pathophysiology of vivax malaria. Trends Parasitol 2009, 25:220-227.

5. Barcus MJ, Basri H, Picarima H, Manyakori C, Sekartuti, Elyazar I, Bangs MJ Maguire JD, Baird JK: Demographic risk factors for severe and fatal vivax and falciparum malaria among hospital admissions in northeastern Indonesian Papua. Am J Trop Med Hyg 2007, 77:984-991.

6. Karyana M, Burdarm L, Yeung S, Kenangalem E, Wariker N, Maristela R, Umana KG, Vemuri R, Okoseray MJ, Penttinen PM, Ebsworth P, Sugiarto P, Anstey NM, Tjitra E, Price RN: Malaria morbidity in Papua Indonesia, an area with multidrug resistant Plasmodium vivax and Plasmodium falciparum. Malar J 2008, 7:148.

7. Kochar DK, Saxena V, Singh N, Kochar SK, Kumar SV, Das A: Plasmodium vivax malaria. Emerg Infect Dis 2005, 11:132-134.

8. Price RN, Tjitra E, Guerra CA, Yeung S, White NJ, Anstey NM: Vivax malaria: neglected and not benign. Am J Trop Med Hyg 2007, 77:79-87.

9. Tjitra E, Anstey NM, Sugiarto P, Warikar N, Kenangalem E, Karyana M, Lampah DA, Price RN: Multidrug-resistant Plasmodium vivax associated with severe and fatal malaria: a prospective study in Papua, Indonesia. PLoS Med 2008, 5:e128.

10. Genton B, D'Acremont V, Rare L, Baea K, Reeder JC, Alpers MP, Muller Plasmodium vivax and mixed infections are associated with severe malaria in children: a prospective cohort study from Papua New Guinea. PLOS Med 2008, 5:e127.

11. Poespoprodjo JR, Fobia W, Kenangalem E, Lampah DA, Hasanuddin A, Warikar N, Sugiarto P, Tjitra E, Anstey NM, Price RN: Vivax malaria: a major cause of morbidity in early infancy. Clin Infect Dis 2009, 48:1704-1712.

12. Poespoprodjo JR, Fobia W, Kenangalem E, Lampah DA, Warikar N, Seal A, McGready R, Sugiarto P, Tjitra E, Anstey NM, Price RN: Adverse pregnancy outcomes in an area where multidrug-resistant Plasmodium vivax and Plasmodium falciparum infections are endemic. Clin Infect Dis 2008, 46:1374-1381.

13. Baird JK: Eliminating malaria - all of them. Lancet 2010, 376:1883-1885.

14. Bockarie MJ, Dagoro H: Are insecticide-treated bednets more protective against Plasmodium falciparum than Plasmodium vivax-infected mosquitoes? Malar J 2006, 5:15

15. Bousema T, Drakeley C: Epidemiology and infectivity of Plasmodium falciparum and Plasmodium vivax gametocytes in relation to malaria control and elimination. Clin Microbiol Rev 2011, 24:377-410.

16. Luxemburger C, Perea WA, Delmas G, Pruja C, Pecoul B, Moren A: Permethrin-impregnated bed nets for the prevention of malaria in schoolchildren on the Thai-Burmese border. Trans $R$ Soc Trop Med Hyg 1994, 88:155-159.

17. Mendis K, Sina BJ, Marchesini P, Carter R: The neglected burden of Plasmodium vivax malaria. Am J Trop Med Hyg 2001, 64:97-106.

18. Sattabongkot J, Tsuboi T, Zollner GE, Sirichaisinthop J, Cui L: Plasmodium vivax transmission: chances for control? Trends Parasitol 2004, 20:192-198.

19. Wells TN, Burrows JN, Baird JK: Targeting the hypnozoite reservoir of Plasmodium vivax: the hidden obstacle to malaria elimination. Trends Parasitol 2010, 26:145-151.

20. Pampana E: A textbook of malaria eradication. London: Oxford University Press; 1969.

21. Tatem AJ, Smith DL, Gething PW, Kabaria CW, Snow RW, Hay SI: Ranking of elimination feasibility between malaria-endemic countries. Lancet 2010 376:1579-1591.

22. McKenzie FE, Jeffery GM, Collins WE: Plasmodium vivax blood-stage dynamics. J Parasitol 2002, 88:521-535.

23. Awab GR, Pukrittayakamee S, Imwong M, Dondorp AM, Woodrow CJ, Lee SJ, Day NP, Singhasivanon P, White NJ, Kaker F: Dihydroartemisininpiperaquine versus chloroquine to treat vivax malaria in Afghanistan: an open randomized, non-inferiority, trial. Malar J 2010, 9:105.

24. Douglas NM, Anstey NM, Angus BJ, Nosten F, Price RN: Artemisinin combination therapy for vivax malaria. Lancet Infect Dis 2010, 10:405-416.
25. Ratcliff A, Siswantoro H, Kenangalem E, Maristela R, Wuwung RM, Laihad F, Ebsworth EP, Anstey NM, Tjitra E, Price RN: Two fixed-dose artemisinin combinations for drug-resistant falciparum and vivax malaria in Papua, Indonesia: an open-label randomised comparison. Lancet 2007, 369:757-765

26. Boyd MF, Kitchen SF: On the infectiousness of patients infected with Plasmodium vivax and Plasmodium falciparum. Am J Trop Med Hyg 1937, s1-17:253-262.

27. Collins WE, Sullivan JS, Nace D, Williams T, Sullivan JJ, Galland GG, Grady KK, Bounngaseng A: Experimental infection of Anopheles farauti with different species of Plasmodium. J Parasitol 2002, 88:295-298.

28. Gething PW, Van Boeckel TP, Smith DL, Guerra CA, Patil AP, Snow RW, Hay SI: Modelling the global constraints of temperature on transmission of Plasmodium falciparum and P. vivax. Parasit Vectors 2011, 4:92.

29. Coatney GR: Relapse in malaria - an enigma. J Parasitol 1976, 62:3-9.

30. Garnham PC: Malaria parasites of man: life-cycles and morphology (excluding ultrastructure). In Malaria: Principles and practice of malariology. Edinburgh, UK: Churchill Livingstone; 1989:61-96.

31. Krotoski WA: The hypnozoite and malarial relapse. Prog Clin Parasitol 1989, 1:1-19.

32. Baird JK, Hoffman SL: Primaquine therapy for malaria. Clin Infect Dis 2004 39:1336-1345.

33. Galappaththy GN, Omari AA, Tharyan P: Primaquine for preventing relapses in people with Plasmodium vivax malaria. Cochrane Database Syst Rev 2007, 1:CD004389.

34. Anthony MP, Burrows JN, Duparc S, Moehrle JJ, Wells TN: The global pipeline of new medicines for the control and elimination of malaria. Malar J 2012, 11:316.

35. Beutler E: G6PD deficiency. Blood 1994, 84:3613-3636

36. Cappellini MD, Fiorelli G: Glucose-6-phosphate dehydrogenase deficiency. Lancet 2008, 371:64-74.

37. WHO: Guidlines for the treatment of malaria, Second Edition. Geneva, Switzerland: World Health Organization; 2010.

38. Hay SI, Okiro EA, Gething PW, Patil AP, Tatem AJ, Guerra CA, Snow RW: Estimating the global clinical burden of Plasmodium falciparum malaria in 2007. PLoS Med 2010, 7:e1000290.

39. Patil AP, Okiro EA, Gething PW, Guerra CA, Sharma SK, Snow RW, Hay SI: Defining the relationship between Plasmodium falciparum parasite rate and clinical disease: statistical models for disease burden estimation. Malar J 2009, 8:186.

40. Coatney GR, Cooper WC: Recrudescence and relapse in vivax malaria. Proceedings of the 4th International Congress on Tropical Medicine 1948, 1:629-639.

41. Garnham PC, Bray RS, Bruce-Chwatt L, Draper CC, Killick-Kendrick R, Sergiev PG, Tiburskaja NA, Shute PG, Maryon M: A strain of Plasmodium vivax characterized by prolonged incubation: morphological and biological characteristics. Bull World Health Organ 1975, 52:21-32.

42. Winckel CWF: Long latency in Plasmodium vivax infections in a temperate zone. Doc Med Geog Trop 1955, 7:292-298.

43. Coatney GR, Collins WE, Contacos PG: Plasmodium vivax. In The Primate Malarias. Bethesda, MD: U.S. National Institute of Allergy and Infectious Diseases; 1971:43-67

44. Mueller I, Galinski MR, Baird JK, Carlton JM, Kochar DK, Alonso PL, Del Portillo HA: Key gaps in the knowledge of Plasmodium vivax, a neglected human malaria parasite. Lancet Infect Dis 2009, 9:555-566.

45. Baird JK, Rieckmann KH: Can primaquine therapy for vivax malaria be improved? Trends Parasitol 2003, 19:115-120.

46. Shute PG, Lupascu G, Branzei P, Maryon M, Constantinescu P, Bruce-Chwatt U, Draper CC, Killick-Kendrick R, Garnham PC: A strain of Plasmodium vivax characterized by prolonged incubation: the effect of numbers of sporozoites on the length of the prepatent period. Trans $R$ Soc Trop Med Hyg 1976, 70:474-481.

47. White NJ: Determinants of relapse periodicity in Plasmodium vivax malaria. Malar J 2011, 10:297.

48. Douglas NM, Nosten F, Ashley EA, Phaiphun L, Van Vugt M, Singhasivanon $P$, White NJ, Price RN: Plasmodium vivax recurrence following falciparum and mixed species malaria: risk factors and effect of antimalarial kinetics. Clin Infect Dis 2011, 52:612-620.

49. Shanks GD, White NJ: The activation of vivax malaria hypnozoites by infectious diseases. Lancet Infect Dis 2013, 13:900-906.

50. Lysenko AJ, Semashko IN: Geography of malaria. A medico-geographic profile of an ancient disease. In Itogi Nauki: Medicinskaja Geografija. Edited by Lebedew AW. Moscow: Academy of Sciences, USSR; 1968:25-146. 
51. Lover AA, Coker RJ: Quantifying effect of geographic location on epidemiology of Plasmodium vivax malaria. Emerg Infect Dis 2013, 19:1058-1065

52. Macdonald G: The Epidemiology and Control of Malaria. London: Oxford University Press; 1957.

53. Holt B, Lessard JP, Borregaard MK, Fritz SA, Araujo MB, Dimitrov D, Fabre PH Graham CH, Graves GR, Jonsson KA, Nogues-Bravo D, Wang ZH, Whittaker RJ, Fjeldsa J, Rahbek C: An update of Wallace's zoogeographic regions of the world. Science 2013, 339:74-78.

54. Peel MC, Finlayson BL, McMahon TA: Updated world map of the KoppenGeiger climate classification. Hydrol Earth Sys Sci 2007, 11:1633-1644.

55. Olson DM, Dinerstein E, Wikramanayake ED, Burgess ND, Powell GVN, Underwood EC, D'Amico JA, Itoua I, Strand HE, Morrison JC, Loucks CJ, Allnutt TF, Ricketts TH, Kura Y, Lamoreux JF, Wettengel WW, Hedao P, Kassem KR: Terrestrial ecoregions of the worlds: a new map of life on Earth. Bioscience 2001, 51:933-938.

56. PubMed. http://www.ncbi.nlm.nih.gov/pubmed/.

57. Howes RE, Battle KE, Satyagraha AW, Baird JK, Hay SI: G6PD deficiency: global distribution, genetic variants and primaquine therapy. Adv Parasitol 2013, 81:133-201.

58. Cooper WC, Myatt AV, Hernandez T, Jeffery GM, Coatney GR: Studies in human malaria. XXXI. Comparison of primaquine, isopentaquine, SN3883, and pamaquine as curative agents against Chesson strain vivax malaria. Am J Trop Med Hyg 1953, 2:949-957.

59. Gogtay NJ, Desai S, Kamtekar KD, Kadam VS, Dalvi SS, Kshirsagar NA: Efficacies of 5- and 14-day primaquine regimens in the prevention of relapses in Plasmodium vivax infections. Ann Trop Med Parasitol 1999 93:809-812.

60. Alvarez G, Pineros JG, Tobon A, Rios A, Maestre A, Blair S, Carmona-Fonseca $\mathrm{J}$ : Efficacy of three chloroquine-primaquine regimens for treatment of Plasmodium vivax malaria in Colombia. Am J Trop Med Hyg 2006, 75:605-609.

61. Looareesuwan S, White NJ, Chittamas S, Bunnag D, Harinasuta T: High rate of Plasmodium vivax relapse following treatment of falciparum malaria in Thailand. Lancet 1987, 2:1052-1055.

62. Taggart JV, Earle DP Jr, Berliner RW, Welch WJ, Zubrod CG, Jailer JW, Kuhn $\mathrm{BH}$, Norwood J, Shannon JA: Studies on the chemotherapy of the human malarias; the antimalarial activity of quinacrine. J Clin Invest 1948, 27:93-97.

63. Baird JK: Chloroquine resistance in Plasmodium vivax. Antimicrob Agents Chemother 2004, 48:4075-4083.

64. Guerra CA, Hay SI, Lucioparedes LS, Gikandi PW, Tatem AJ, Noor AM, Snow RW: Assembling a global database of malaria parasite prevalence for the Malaria Atlas Project. Malar J 2007, 6:17.

65. Google Maps. https://maps.google.com/.

66. ESRI: ArcG/S Desktop 10.0. Redlands, CA: Environmental Systems Resource Institute; 2010

67. Viechtbauer $\mathrm{W}$ : Bias and efficiency of meta-analytic variance estimators in the random-effects model. J Educ Behav Stat 2005, 30:261-293.

68. Viechtbauer W: Conducting meta-analyses in $\mathrm{R}$ with the metafor package. Journal of Statistical Software 2010, 36:1-48.

69. R Core Team: R: A Language and Environment for Statistical Computing. Vienna, Austria: R Foundation for Statistical Computing; 2013.

70. Raudenbush SW: Analyzing effect sizes: random effects models. In The handbook of research synthesis and meta-analysis. 2nd edition. Edited by Cooper H, Hedges LV, Valentine JC. New York: Russell Sage Foundation; 2009:295-315.

71. Freeman MF, Tukey JW: Transformations related to the angular and the square root. Ann Math Stat 1950, 21:607-611.

72. Clayton D, Hills M: Statistical Models in Epidemiology. Oxford, UK: Oxford University Press; 2013.

73. Ulm K: A simple method to calculate the confidence interval of a standardized mortality ratio (SMR). Am J Epidemiol 1990, 131:373-375.

74. Gonzalez-Ceron L, Mu J, Santillan F, Joy D, Sandoval MA, Camas G, Su X Choy EV, Torreblanca R: Molecular and epidemiological characterization of Plasmodium vivax recurrent infections in southern Mexico. Parasit Vectors 2013, 6:109.

75. Llanos-Cuentas A, Lacerda MV, Rueangweerayut R, Krudsood S, Gupta SK, Kochar SK, Arthur P, Chuenchom N, Mohrle JJ, Duparc S, Ugwuegbulam C, Kleim JP, Carter N, Green JA, Kellam L: Tafenoquine plus chloroquine for the treatment and relapse prevention of Plasmodium vivax malaria
(DETECTIVE): a multicentre, double-blind, randomised, phase $2 \mathrm{~b}$ doseselection study. Lancet 2014, 383:1049-1058.

76. Sharma SN, Subbarao SK, Choudhury DS, Pandey KC: Role of An. culicifacies and An. stephensi in malaria transmission in urban Delhi. Indian J Malariol 1993, 30:155-168.

77. Sinka ME, Bangs MJ, Manguin S, Chareonviriyaphap T, Patil AP, Temperley WH, Gething PW, Elyazar IR, Kabaria CW, Harbach RE, Hay SI: The dominant Anopheles vectors of human malaria in the Asia-Pacific region: occurrence data, distribution maps and bionomic precis. Parasit Vectors 2011, 4:89.

78. Taylor JE, Pacheco MA, Bacon DJ, Beg MA, Machado RL, Fairhurst RM, Herrera S, Kim JY, Menard D, Povoa MM, Villegas L, Mulyanto, Snounou G, Cui L, Zeyrek FY, Escalante AA: The evolutionary history of Plasmodium vivax as inferred from mitochondrial genomes: parasite genetic diversity in the Americas. Mol Biol Evol 2013, 30:2050-2064.

79. Arnold J, Alving AS, Hockwald RS, Clayman CB, Dern RJ, Beutler E: Natural history of Korean vivax malaria after deliberate inoculation of human volunteers. J Lab Clin Med 1954, 44:723-726.

80. Cooper WC, Ruhe DS, Coatney CR, Josephson ES, Young MD: Studies in human malaria. VIII. The protective and therapeutic action of quinacrine against St. Elizabeth strain vivax malaria Am J Hyg 1949, 49:25-40.

81. Tiburskaja NA, Vrublevskaja OS: The course of infection caused by the North Korean strain of Plasmodium vivax. In WHO Malaria Report 1977 1977, 895:1-19. 1-19.

82. Mueller I, Galinski MR, Tsuboi T, Arevalo-Herrera M, Collins WE, King CL: Natural acquisition of immunity to Plasmodium vivax: epidemiological observations and potential targets. Adv Parasitol 2013, 81:77-131.

83. Bignami $\mathrm{A}$ : Concerning the pathogenesis of relapses in malarial fevers. South Med J 1913, 6:79-89.

84. Price RN, Douglas NM, Anstey NM: New developments in Plasmodium vivax malaria: severe disease and the rise of chloroquine resistance. Curr Opin Infect Dis 2009, 22:430-435.

85. Rieckmann KH, Davis DR, Hutton DC: Plasmodium vivax resistance to chloroquine? Lancet 1989, 2:1183-1184.

86. Boyd MF: The influence of sporozoite dosage in vivax malaria. Am J Trop M 1940, 20:279-286.

87. Coatney GR, Cooper WC, YM D, Burgess RW: Studies in human malaria. XVIII. The life pattern of sporozoite-induced St Elizabeth strain vivax malaria. Am J Epidemio/ 1950, 51:200-215.

88. Findlay GM: Recent advances in chemotherapy. Philadelphia, USA: The Blakiston Company; 1951

89. Most $\mathrm{H}$ : Clinical trials of anti-malarial drugs. In Internal Medicine in World War II, Infectious Diseases, vol 2. Volume 2. Washington, D.C.: Office of the Surgeon General, Department of the Army; 1963:479-492.

90. Coatney GR, Alving AS, Jones R Jr, Hankey DD, Robinson DH, Garrison PL, Coker WG, Donovan WN, Di Lorenzo A, Marx RL, Simmons IH: Korean vivax malaria. V. Cure of the infection by primaquine administered during long-term latency. Am J Trop Med Hyg 1953, 2:985-988.

doi:10.1186/1475-2875-13-144

Cite this article as: Battle et al:: Geographical variation in Plasmodium vivax relapse. Malaria Journal 2014 13:144.

\section{Submit your next manuscript to BioMed Central and take full advantage of:}

- Convenient online submission

- Thorough peer review

- No space constraints or color figure charges

- Immediate publication on acceptance

- Inclusion in PubMed, CAS, Scopus and Google Scholar

- Research which is freely available for redistribution 